\title{
Number of blastocysts biopsied as a predictive indicator to obtain at least one normal/balanced embryo following preimplantation genetic diagnosis with single nucleotide polymorphism microarray in translocation cases
}

\author{
Yi-zi Wang ${ }^{1,2}$ • Chen-hui Ding ${ }^{1,2}$ • Jing Wang ${ }^{1,2}$ - Yan-hong Zeng ${ }^{1,2}$ • Wen Zhou ${ }^{1,2}$ • \\ Rong $\mathrm{Li}^{1,2}$ • Can-quan Zhou ${ }^{1,2} \cdot$ Ming-Fen Deng ${ }^{1,2} \cdot$ Yan-wen $\mathrm{Xu}^{1,2}$
}

Received: 21 April 2016/Accepted: 23 October 2016/Published online: 7 November 2016

(C) The Author(s) 2016. This article is published with open access at Springerlink.com

\begin{abstract}
Purpose The aim of this study is to investigate the minimum number of blastocysts for biopsy to increase the likelihood of obtaining at least one normal/balanced embryo in preimplantation genetic diagnosis (PGD) for translocation carriers.

Methods This blinded retrospective study included 55 PGD cycles for Robertsonian translocation (RT) and 181 cycles for reciprocal translocation ( $\mathrm{rcp}$ ) to indicate when only one of the couples carried a translocation. Single-nucleotide polymorphism microarray after trophectoderm biopsy was performed. Results Reliable results were obtained for 355/379 (93.7 \%) biopsied blastocysts in RT group and 986/1053 (93.6 \%) in rcp group. Mean numbers of biopsied embryos per patient, normal/balanced embryos per patient, and mean normal/
\end{abstract}

Capsule To obtain at least one normal/balanced embryo, the number of blastocysts biopsied should be $\geq 2$ and $\geq 4$ for Robertsonian and reciprocal translocation carriers.

Yi-zi Wang and Chen-hui Ding contributed equally to this work.

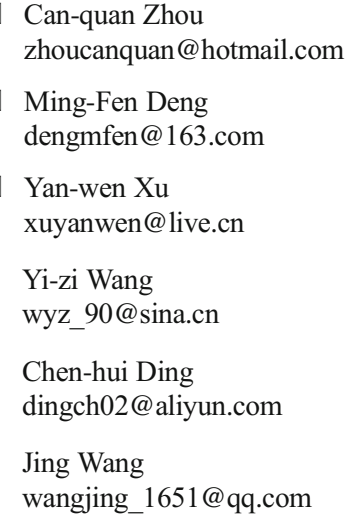

balanced embryo rate per patient were $7.4,3.1$, and $40.7 \%$ in RT group and 8.0, 2.1, and $27.3 \%$, respectively, in rep group. In a regression model, three factors significantly affected the number of genetically transferrable embryos: number of biopsied embryos $(P=0.001)$, basal FSH level $(P=0.040)$, and maternal age $(P=0.027)$. ROC analysis with a cutoff of 1.5 was calculated for the number of biopsied embryos required to obtain at least one normal/balanced embryo for RT carriers. For rcp carriers, the cutoff was 3.5. The clinical pregnancy rate per embryo transfer was 44.2 and $42.6 \%$ in RT and rcp groups $(P=0.836)$.

Conclusions The minimum numbers of blastocysts to obtain at least one normal/balanced embryo for RT and rcp were 2 and 4 under the conditions of female age $<37$ years with a basal FSH level < 11.4 IU/L.
Yan-hong Zeng
792013240@qq.com
Wen Zhou
zhouw@126.com
Rong Li
287344897@qq.com

1 Reproductive Medical Center, The First Affiliated Hospital of Sun Yat-sen University, (Floor 1, Building 6th) ZhongshanEr Road No. 58, Yuexiu District, Guangzhou, Guangdong 510080, China

2 Guangdong Provincial Key Laboratory of Reproductive Medicine, Guangzhou, China 
Keywords Robertsonian translocation $\cdot$ Reciprocal translocation · Preimplantation genetic diagnosis . Single-nucleotide polymorphism · Trophectoderm biopsy

\section{Introduction}

Translocation is one of the most common structural rearrangements of chromosomes, with an estimated 0.4 and $0.19 \%$ prevalence in prenatal samples and newborns [1]. Robertsonian translocation (RT) and reciprocal translocation (rcp) usually result in no obvious phenotypic abnormalities when balanced. However, both types of translocation carriers have a high rate of producing a chromosomally unbalanced gamete, which results in a high risk for miscarriage. For a RT carrier, depending on the segregation patterns in meiotic divisions, only $1 / 6$ normal and 1/6 balanced gametes are alternately segregated, which can produce normal or balanced (normal/balanced) embryos [2]. For rcp carriers, the alternate gametes merely account for $1 / 9$ [3]. The risk of miscarriage is estimated at 20-33\% in couples who carry RT and 47-53\% in couples who carry rcp [4].

Preimplantation genetic diagnosis (PGD) is used as an alternative for carriers of balanced translocation to select normal/balanced embryos for transfer to minimize the risk of miscarriage due to abnormal segregation of the translocation and to maximize the chance of a healthy child. Recently, a variety of biopsy methods combined with different biopsy stages and modes of embryo transfer have been attempted by PGD. In the PGD field, multiple displacement amplification (MDA)-based single-nucleotide polymorphism (SNP) arrays have been commonly utilized to discriminate euploid embryos from aneuploid embryos [5]. More recently, several polymerase chain reaction-based next-generation sequencing protocols have been developed and validated [6-8]. Both platforms can detect expected unbalanced segments arising from translocation chromosomes and distinguish aneuploidies from other chromosomes. In our center, SNP arrays are routinely used. In addition, blastocyst biopsy rather than cleavage-stage biopsy is now generally recommended because it has the advantage of a lower mosaic rate, reduced biopsy-related damage, and higher developmental potential [9].

With the advancement of diagnostic technology, PGD results for translocations have become more accurate. However, not every PGD patient has an adequate number of blastocysts for biopsy, and some patients may not have normal/balanced embryo for transfer even though they have several blastocysts for PGD. The multi-center analysis from the European Society of Human Reproduction and Embryology (ESHRE) consortium reported that only $26 \%$ of successfully diagnosed embryos biopsied from translocation carriers were suitable for transfer [10]. The normal/balanced embryo rate (transferrable rate) for a RT male carrier, RT female carrier, rcp male carrier, and rcp female carrier were $38,29,22$, and $20 \%$, respectively
[11]. However, when the number of transferrable embryos is estimated according to the number of SNP array-biopsied blastocysts, or when determining the minimum number of blastocysts required by biopsy to obtain at least one normal/ balanced embryo for carriers, these general data have limited values. The aim of this study was to investigate the minimum number of blastocysts required for PGD using a SNP array to maximize the chance of obtaining at least one normal/ balanced blastocyst for transfer.

\section{Materials and methods}

\section{Patient information}

In this study, 51 patients with an indication of RT underwent 55 cycles of PGD and 131 couples with an indication of rcp underwent 181 cycles of PGD from July 2013 to December 2014. Translocation status was confirmed by conventional karyotype analysis. There were 16 couples involving female carriers and 35 couples involving male carriers in the RT group, and 67 couples involving female carriers and 64 couples involving male carriers in the rcp group. No compound translocation cases were recruited in either group.

\section{Ethical considerations}

Written informed consent describing the limitations, advantages, and necessities of PGD was obtained from participants before couples underwent controlled ovarian stimulation (COS) cycles. All materials and diagnosis protocols in this study were approved by the Faculty of Medical Research Service Ethics Committee, the First Affiliated Hospital of Sun Yat-sen University, China.

\section{Controlled ovarian stimulation and in vitro fertilization}

To obtain an ideal number of oocytes, flexible gonadotropinreleasing hormone $(\mathrm{GnRH})$ agonist long protocols were performed. The starting doses of recombinant follicle-stimulating hormone (FSH) (Gonal-F, Merck-Serono, Geneva, Switzerland; Puregon, NV Organon, Oss, The Netherlands) and/or human menopausal gonadotrophins (HMG, Lizhu, China) were determined experientially according to patient age, body mass index (BMI), basal FSH, antral follicle count (AFC), and/or previous response to ovarian stimulation. According to the patient's ovarian response, monitored by regular transvaginal ultrasound and plasma estradiol levels when necessary, the dose of recombinant FSH and/or HMG was adjusted individually. Oocyte retrieval (OR) through vaginal puncture under ultrasound guidance was performed 34 $36 \mathrm{~h}$ after the administration of human chorionic gonadotropin (250 $\mu \mathrm{g}$ Ovidrel $^{\mathrm{R}}$; EMD-Serono). Only meiosis II (MII) stage 
oocytes were microinjected by ICSI $4-6 \mathrm{~h}$ after OR. A fertilization check was performed $16-18 \mathrm{~h}$ post-ICSI.

Three females in the RT group and 44 females in the rcp group received multiple controlled ovarian stimulations to accumulate a pool of embryos for testing. The number of stimulation cycles ranged from one to four.

\section{Embryo culture and biopsy}

All embryos were cultured in sequential media (G1 and G2, Vitrolife, Goteborg, Sweden) to blastocyst stage under $6 \%$ $\mathrm{CO}_{2}, 5 \% \mathrm{O}_{2}$, and $89 \% \mathrm{~N}_{2}$ in a COOK mini-incubator (Bloomington, IN, USA) for further manipulation. An 1820-mm hole was then made by laser drilling in the zona pellucida of all embryos with no sign of hatching on the morning of day 5 (D5). Blastocysts with trophectoderm (TE) cells herniating out of the zona pellucida were chosen for biopsy on D5 or Day 6 (D6). Approximately 5-10 TE cells were aspirated with a biopsy pipette (internal diameter $30 \mu \mathrm{m}$ ) and dissected with an OctaxShot ${ }^{\mathrm{TM}}$ laser system. Biopsied TE cells were washed three times in G-MOPS medium (Vitrolife) and then either used directly for whole genome amplification (WGA) or stored at $-20^{\circ} \mathrm{C}$ for future WGA.

\section{Blastocyst vitrification and thawing}

Blastocysts were vitrified after biopsy using a Kitazato vitrification kit (KitazatoBiopharma Co. Ltd., Shizuoka, Japan) in combination with Vitrification Cryotop. Each blastocyst was stored in an individual straw. The vitrification and thawing procedure was carried out according to the protocol recommended by the Kitazato vitrification kit.

\section{SNP microarray analysis}

All biopsied TE cells were placed in $5 \mu$ l of DNA stabilizing buffer $(0.2 \mathrm{M} \mathrm{KOH})$ for SNP microarrays. The cell samples from each embryo first underwent cell lysis and the WGA protocol. For SNP arrays, cells were lysed using an alkaline denaturation buffer $(0.2 \mathrm{M} \mathrm{NaOH})$ followed by a 4-h modified MDA protocol using $\varphi 29$ polymerase to generate template DNA. Then, $4 \mu \mathrm{L}(200 \mathrm{ng})$ of DNA product was used in a 13-hour WGA amplification protocol using $\varphi 29$ polymerase. Each DNA product then underwent enzymatic end-point fragmentation and the resuspended DNA samples were then dispensed onto Human CytoSNP-12 DNA analysis bead chips (Illumnia, San Diego, CA) and allowed to hybridize for $12 \mathrm{~h}$. Each CytoSNP-12 bead chip contained approximately 301,000 SNPs and other genetic markers. Stringency washes were performed to remove un-hybridized and non-specific bound DNA. Bead chips dried in a desiccator were scanned using an Illumina iScan Bead Array Reader. Raw data analysis was accomplished using Illumina Genome Studio software.
One of the limitations of the SNP array is the inability to distinguish a balanced embryo from a normal embryo.

\section{Embryo transfer and luteal support}

Only blastocysts with concordant normal/balanced results were considered for embryo transfer. According to the patients' menstruation characteristics, the natural ovulation cycle (NC) or hormone replacement treatment protocol (HRT) was used for endometrial preparation. Six days after observing a surge in serum luteinizing hormone or progesterone administration, qualified blastocysts, prioritized based on the best quality before biopsy, were thawed. Re-expanded blastocysts were transferred 3-4 h after warming. Individual luteal support protocols were administered. No more than two blastocysts were transferred.

\section{Outcome parameters}

All parameters were classified according to the type of translocation. Patient baseline characteristics and COS parameter are presented in Table 1. Transferrable rate was calculated according to the equation: number of normal/balanced embryos/number of embryos biopsied and was the main PGD outcome. Biopsy outcomes of SNP arrays are summarized in Table 2. Pregnancy was confirmed 12 days after embryo transfer (ET) if the serum HCG was elevated. Clinical pregnancy was defined as the presence of fetal heartbeat (FHB) in the uterus under transvaginal ultrasound scan.

\section{Statistical analysis}

All statistical procedures were conducted with SPSS version 22.0 software (SPSS Inc., Chicago, IL, USA). A combination of the Student's $t$ test, F test for variance, and chi-square test were performed. To determine correlative factors, a linear regression model $\left(\mathrm{Y}=\mathrm{B}_{0}+\mathrm{B}_{1} \mathrm{X}_{1}+\mathrm{B}_{2} \mathrm{X}_{2}\right)$ was used to analyze the entire data pooled from the two groups. Different associated variables (or prognostic factors) such as age, BMI, basal FSH, basal $E_{2}$ level, total amount of Gn, $E_{2}$ level on HCG day, number of oocytes retrieved, and number of biopsied blastocysts were entered as independent variables and the number of genetically normal/balanced blastocysts after PGD was regarded as a dependent variable. ROC curves were calculated to determine the cutoff value for the number of biopsied blastocysts with the aim of obtaining at least one normal/balanced blastocyst. $P$ values $<0.05$ were considered significant.

\section{Results}

Fifty-one patients underwent 55 PGD cycles for RT and 131 couples underwent 181 cycles of PGD for rcp. To increase the 
Table 1 COS parameters and results

\begin{tabular}{lll}
\hline & $\begin{array}{l}\text { Robertsonian } \\
\text { translocation }\end{array}$ & $\begin{array}{l}\text { Reciprocal } \\
\text { translocation }\end{array}$ \\
\hline Couples & 51 & 131 \\
Female carriers & 16 & 67 \\
Male carriers & 35 & 64 \\
COS cycle & 55 & 181 \\
Patients with one stimulation cycle & 48 & 86 \\
Patients with $\geq 2$ stimulation cycles & 3 & 45 \\
Average maternal age (years) & $31.2 \pm 3.7$ & $30.7 \pm 3.9$ \\
Maternal BMI (kg/m ${ }^{2}$ ) & $20.8 \pm 3.0$ & $21.2 \pm 4.1$ \\
Basal FSH (IU/L) & $5.7 \pm 1.8$ & $5.7 \pm 1.7$ \\
Basal E (pg/ml) & $34.4 \pm 15.8$ & $36.0 \pm 16.1$ \\
Initial dose of Gn (IU) & $196.2 \pm 61.4$ & $198.9 \pm 56.6$ \\
Stimulation length (days) & $11.2 \pm 1.7$ & $11.2 \pm 2.0$ \\
Total amount of Gn (IU) & $2337.3 \pm 913.8$ & $2396.7 \pm 878.4$ \\
HCG day E2 (pg/ml) & $3207 \pm 1429.5$ & $2891 \pm 1403.8$ \\
Retrieved oocytes/OPU & $19.8 \pm 8.7^{*}$ & $17.0 \pm 8.2^{*}$ \\
MII & $16.4 \pm 7.1^{*}$ & $14.0 \pm 6.6^{*}$ \\
2PN (fertilized) & $12.6 \pm 5.7$ & $11.6 \pm 5.6$ \\
Cleavage rate/\% & $94.8 \pm 8.3$ & $96.5 \pm 10.2$ \\
Mean number of embryos biopsied & $7.4 \pm 3.6$ & $8.0 \pm 3.5$ \\
Number of normal/balanced & $3.1 \pm 2.2^{*}$ & $2.1 \pm 1.6^{*}$ \\
$\quad$ embryos & & 16 \\
Couples without Normal/balanced & 4 & \\
embryos for FET & & \\
\hline & & \\
\hline
\end{tabular}

Data are expressed as mean \pm SD. Data refer to analyzed cycles that were only those who were completed; cancelled cycles are not included in the analysis

$\mathrm{COH}$ controlled ovarian hyperstimulation, $\mathrm{Gn}$ gonadotropins, $O P U$ oocyte pick-up, $M I I$ metaphase II, FET frozen embryo transfer

${ }^{*} P<0.05$; significant difference between Robertsonian translocation group and reciprocal translocation group (Welch $t$ test with $\mathrm{F}$ test for variance performed as appropriate)

number of embryos for PGD due to low ovarian reserve or poor response, multiple ovarian stimulation treatments $(2-4$ cycles) were performed for 3 RT carriers and 45 rcp patients. There was no difference in the mean maternal age in the RT and rcp groups ( $31.2 \pm 3.7$ and $30.7 \pm 3.9$ years, respectively). Other factors closely associated with the number of biopsy blastocysts are shown in Table 1. There were more retrieved and matured oocytes in the RT group compared with the rcp group $(P<0.05)$; however, the number of normally fertilized embryos and cleavage rate were similar in the two groups. The number of normal/balanced blastocysts was significantly higher for RT carriers compared with rep carriers $(P<0.05)$, although the number of blastocysts available for biopsy was the same.

The outcomes of biopsied blastocysts are shown in Table 2 . MDA-based WGA was processed successfully for 1341 samples. Of these, 355 were from the RT group and 986 were from the rcp group. The failure or "no result" rate was similar between the two groups. Poor embryo quality was the main factor attributed to amplification failure.

Among the diagnosed blastocysts, 160 (42.2\%) were normal/balanced with no aneuploidy in non-translocated chromosomes in the RT group, $105(27.7 \%)$ were balanced but with aneuploidy of non-translocated chromosomes, $72(19 \%)$ were unbalanced only in the translocated chromosomes, and $18(4.7 \%)$ were unbalanced along with abnormalities in other chromosomes. For RT carriers, the mean detection rate of transferrable blastocysts was $40.7 \%$ (95\% confidence interval [CI], $34.6-46.9 \%)$.

In the rcp group, $283(26.9 \%)$ diagnosed blastocysts were normal/balanced with no aneuploidy in non-translocated chromosomes, $182(17.3 \%)$ blastocysts were balanced with aneuploidy in non-translocated chromosomes, $425(40.4 \%)$ of diagnosed blastocysts were unbalanced only in translocated chromosomes, and 96 (9.1\%) embryos were unbalanced with abnormalities in other chromosomes. For rcp carriers, the mean detection rate of transferrable blastocysts was $27.3 \%$ (95\%CI, 23.9-30.7\%).

The rates for unbalanced translocation with or without sporadic aneuploidy were significantly higher for reciprocal translocation carriers $(P<0.01)$. Therefore, a significantly higher rate of unbalanced translocation was detected in the rcp group compared with the RT group. This translated into a comparatively lower detection rate of transferrable blastocysts for rcp carriers compared with RT carriers. A comparison of the detection rates in the two groups was statistically significant $(P<0.01)$.

Data was merged from the two groups and a linear regression model was used to examine exploratory variables such as age, BMI, basal FSH, basal $\mathrm{E}_{2}$ level, total amount of $\mathrm{Gn}, \mathrm{E}_{2}$ on HCG day, number of oocytes retrieved, and number of biopsied blastocysts to number of genetically normal/ balanced blastocysts after PGD. We identified three significant factors that affected the number of genetically transferrable embryos in the adjusted model: number of biopsied embryos (coefficient: $0.489,95 \% \mathrm{CI} 0.350-0.557, P=0.001$ ), basal FSH level (bFSH) (coefficient: 0.138, $95 \%$ CI $0.071-$ $0.225, P=0.040$ ), and maternal age (mAge) (coefficient: $0.160,95 \%$ CI $0.089-0.201, P=0.027$ ). According to mathematical calculations, this significant impact only existed if the inequality was satisfied: $0.16^{*} \mathrm{mAge}+0.138^{*} \mathrm{bFSH} \leq 7.5$.

Because basal FSH levels were below 11.4 IU/L in all cases, a subgroup excluding females aged over 37 (4 couples in the RT group and 12 couples in the rcp group, $21 \mathrm{COS}$ cycles totally) was created for further ROC analysis (Fig. 1). A positive result was defined as finding at least one normal/ balanced blastocyst after biopsy. According to ROC curves, a threshold number of 1.5 biopsied blastocysts was identified in RT patients achieving positive results (sensitivity $=86.8 \%$, 
Table 2 Outcomes of biopsied blastocysts

\begin{tabular}{lllll}
\hline $\begin{array}{l}\text { Population } \\
\text { biopsy } \\
\text { results }\end{array}$ & $\begin{array}{l}\text { Robertsonian } \\
\text { Translocation }\end{array}$ & $\begin{array}{l}\text { Reciprocal } \\
\text { Translocation }\end{array}$ & $\begin{array}{l}\text { Maternal } \\
\text { age } \\
\geq 37 \text { years }\end{array}$ & $\begin{array}{l}\text { Maternal } \\
\text { age } \\
<37 \text { years }\end{array}$ \\
\hline $\begin{array}{l}\text { Biopsied embryos } \\
\text { Embryos diagnosed }\end{array}$ & 379 & 1053 & 85 & 1347 \\
$\begin{array}{l}\text { Normal/balanced } \\
\text { translocation without } \\
\text { aneuploidy }\end{array}$ & $160(45.1)^{\mathrm{a}}$ & $986(93.6)$ & $77(90.6)$ & $1264(93.8)$ \\
$\begin{array}{l}\text { Balanced, with other } \\
\text { aneuploidy }\end{array}$ & $105(29.6)^{\mathrm{a}}$ & $283(28.7)^{\mathrm{a}}$ & $17(22.1)^{\mathrm{b}}$ & $426(33.7)^{\mathrm{b}}$ \\
$\begin{array}{l}\text { Unbalanced, without other } \\
\text { aneuploidy }\end{array}$ & $72(20.3)^{\mathrm{a}}$ & $182(18.5)^{\mathrm{a}}$ & $23(29.9)^{\mathrm{b}}$ & 258 \\
$\begin{array}{l}\text { Unbalanced, with other } \\
\text { aneuploidy }\end{array}$ & $18(5.1)^{\mathrm{a}}$ & $425(43.1)^{\mathrm{a}}$ & $25(32.5)^{\mathrm{b}}$ & $473(37.4)^{\mathrm{b}}$ \\
$\begin{array}{l}\text { Embryos undiagnosed } \\
\begin{array}{l}\text { Total abnormality } \\
\text { Mean diagnostic rate of }\end{array}\end{array}$ & $24(6.3)$ & $96(9.7)^{\mathrm{a}}$ & $12(15.6)^{\mathrm{b}}$ & $107(8.5)^{\mathrm{b}}$ \\
$\begin{array}{l}\text { normal/balanced } \\
\text { translocation \% }\end{array}$ & $195(51.4)$ & $67(6.4)$ & $8(9.4)$ & $83(6.2)$ \\
\hline
\end{tabular}

Values are expressed as $n(\%)$ or mean \pm standard deviation of the mean (95\% confidence interval)

${ }^{\text {a }}$ Significant differences $(P<0.05)$ within translocation type (chi-square test): Pearson $=79.63, P<0.001$

${ }^{\mathrm{b}}$ Significant differences $(P<0.05)$ within maternal age (chi-square test): Pearson $=10.69, P=0.014$

${ }^{\mathrm{c}}$ Student's $t$ test: $\mathrm{t}=3.99, P<0.001$

specificity $=66.7 \%)$. For rcp carriers, the threshold number was 3.5 (sensitivity $=90.4 \%$, specificity $=73.3 \%)$. The area under the curve (AUC) in the RT and rcp groups $(0.912$; $95 \%$ CI: $0.812-0.998$ and 0.877 ; $95 \%$ CI: $0.801-0.953$, respectively) indicated that it was a good prognostic test.

Single and double embryo transfers were performed for 139 translocation carriers (41 RT and 98 rcp carriers). Clinical pregnancy was achieved in 33 cases in the RT group and in 54 cases in the rcp group. The clinical pregnancy rate per ET was 44.2 and $42.6 \%$ in the RT group and rcp groups, respectively $(P=0.836)$ (Table 3 ).

Examples of normal and abnormal molecular karyotypes are shown in Fig. 2.

\section{Discussion}

Patients who carry balanced translocations have a high incidence of abnormal meiotic segregations, which decreases the chance of finding an embryo for transfer in carriers. This is exacerbated when carriers also have a poor ovarian reserve. It is critical for the clinic to determine a threshold of the number of blastocysts available to biopsy to maximize the chance of obtaining at least one transferrable blastocyst.

In this study, rep carriers had a significantly higher rate of unbalanced embryos compared with RT carriers. For rcp carriers, only an alternate meiotic segregation pattern can lead to the production of chromosomally normal/balanced gametes, which only accounts for 1/9. For RT patients, the rate of obtaining alternate embryos derived from alternate division is $1 / 3$. The data in the study of Chang et al. of $31.3 \%$ transferrable embryos from 514 biopsied blastomeres was similar to that [12].

However, the normal/balanced rates of $42.2 \%$ in RT and $26.9 \%$ in rcp were both higher than genetically expected in this study. A recent study by Idowu et al. including 539 biopsied embryos used for the same CytoSNP-12 arrays also showed up to $37 \%$ of RT embryos and $19 \%$ of rep embryos were euploid [13]. The reason for a lower transferrable rate in their study might be attributed to two factors. First, nearly half of the embryos $(202 / 539,37.5 \%)$ were from women $\geq 35$ years compared to $24 / 182(13.2 \%)$ in our study. Second, only one

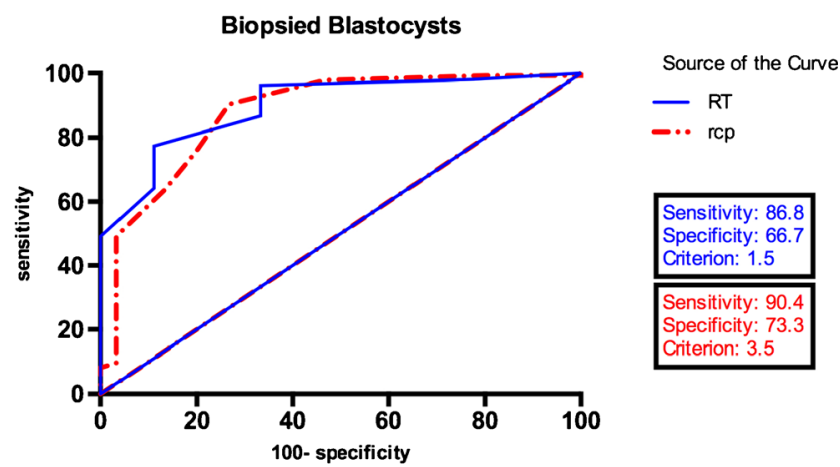

Fig. 1 ROC curves for the relationship between the number of biopsied blastocysts and the outcome of obtaining at least one normal/balanced blastocyst in Robertsonian translocation and reciprocal translocation carriers in subgroups whose basal FSH levels were lower than 11.4 IU/ $\mathrm{L}$ and females who were younger than 37 years of age 
Table 3 Pregnancy outcomes in chromosomal translocation carriers

\begin{tabular}{lll}
\hline & $\begin{array}{l}\text { Robertsonian } \\
\text { translocation }\end{array}$ & $\begin{array}{l}\text { Reciprocal } \\
\text { translocation }\end{array}$ \\
\hline Carriers with no ET & 10 & 33 \\
Carriers with ET & 41 & 98 \\
Cycles with ET & 52 & 169 \\
No. Embryo transferred & 95 & 255 \\
Clinical pregnancy rate per & $44.2^{\mathrm{N}}$ & $42.6^{\mathrm{N}}$ \\
$\quad$ ET, \% & & \\
\hline
\end{tabular}

${ }^{\mathrm{N}}$ No significant difference $(P<0.05)$ between Robertsonian translocation group and reciprocal translocation group (chi-square test): Pearson $=0.043, P=0.836$

fifth of embryos $(102 / 539,18.9 \%)$ was biopsied on D5, and the remaining embryos were biopsied at the cleavage stage. In our study, only trophectoderm biopsy was carried out, and the proportions of D5 and D6 blastocysts biopsied were 957/1432 $(66.8 \%)$ and $475 / 1432(33.2 \%)$, respectively.

The adverse relationship between advanced maternal age and embryo euploidy has been universally accepted [14]. According to our statistical inequality, only when the conditions of female age younger than 37 years with basal FSH levels under $11.4 \mathrm{IU} / \mathrm{L}$ were the conclusions from ROC analysis credible.

The aneuploidy rate was also inversely associated with development stage. Many studies recommend blastocyststage biopsy rather than cleavage-stage biopsy, which has the advantage of reduced biopsy-related damage, lower mosaic rate, and higher pregnancy rate [15-17]. A previous study reported more than half of cleavage stage embryos $(57.7 \%$ of 134 single blastomeres) exhibited mosaicism [18]. Another study evaluated 280 embryos from rcp carriers ( 56 blastocysts and 224 cleavage-stage embryos) and observed that $57.5 \%$ were aneuploid with or without the unbalanced parental translocation. When biopsy only included trophectoderm cells from blastocysts, the aneuploidy prevalence dropped to $26.7 \%$ [19], which was similar to the $26.4 \%$ in the rep group in the current study. The progression of embryos developing from the cleavage stage to the blastocyst stage, referring to blastulation, is clinically important. Because most unbalanced embryos in the cleavage stage degenerated or stopped developing because of chromosomal abnormalities, they did not achieve complete blastulation. Therefore, the finding that the proportion of transferrable embryos tended to increase from the cleavage stage to the blastocyst stage might aid our understanding of this phenomenon. Of note, both D5 and D6 blastocysts were recruited in our study. According to previous studies, embryos that were slower to develop presented with a higher proportion of chromosomal abnormalities compared with embryos that progressed normally [20-22]. Therefore, in our study, we verified that the proportion of normal/balanced blastocysts in D5 blastocysts was significantly higher (318/
908) than that in D6 blastocysts $(125 / 433)(P=0.025)$. Therefore, if the proportion of D5 blastocysts was overwhelming, one normal/balanced blastocyst might be obtained from less biopsied blastocysts.

In the present study, the number of biopsy blastocysts was correlated to transferrable outcomes in a cohort of female patients aged younger than 37 years and with a basal FSH level less than $11.4 \mathrm{IU} / \mathrm{L}$. This study concluded that one normal/ balanced embryo was likely to be obtained from 2 blastocysts in the RT group and 4 blastocysts in the rep group. Median values of biopsied blastocysts (both D5 and D6 recruited) and normal/balanced embryos from a published research study based on SNP were presented as 4, 2 in the RT group and 4, and 1 in the rcp group [23]. This indicated that RT carriers were two times more likely to have a chance of a normal/ balanced embryo than rcp carriers. Indeed, a majority of RT carriers $(94.1 \%)$ and more than half of rep carriers $(65.6 \%)$ in their first COS cycle had blastocyst numbers higher than the threshold calculated here. As for these couples, our findings could be used to predict how many normal/balanced embryos could be obtained if the blastocysts qualifying for biopsy reached a certain number.

This study also demonstrated that $5.9 \%$ of RT couples and $34.4 \%$ of rep patients, diagnosed as having a diminished ovarian reserve or poor response, did not obtain the desired number of blastocysts in their first cycle. In these cases, what clinical strategy should be followed? We share the same opinion with Tulay et al. and Chatziparasidou A et al. [24, 25] who suggested that serial cycles of ovarian stimulation should be undertaken. According to the study of Scriven et al., when each additional embryo was available for biopsy, there was a $13 \%$ higher opportunity of a live birth [26].

To avoid mistakes and cross-contaminations during handling, each SNP chip was only used once for each couple regardless of how many embryos were analyzed in our center. The price of one chip is estimated at US\$3200 in China. If a biopsy is routinely requested after every COS cycle for patients with limited blastocysts, there is likely to be unnecessary expense before obtaining a transferrable blastocyst. In addition, the current policy of China does not allow charging on a per embryo basis. Thus, testing embryos after serial cycles of ovarian stimulation is recommended for couples with limited numbers of blastocysts.

One main limitation of this study was the preprocessing techniques of the SNP chips used for diagnosis. SNP chips avoid many drawbacks inherent in array CGH (which uses ratio labeling), resulting in a lower density and an inability to identify some karyotypes (69, XXX appeared the same as 46, XX) [27]. However, all current techniques have the same limitation, and therefore, it is difficult to distinguish a balanced embryo from a normal embryo unless using parental guidance haplotype analysis. In addition, due to the detection capability of SNP chips, only segmental aneuploidy of no less 
a
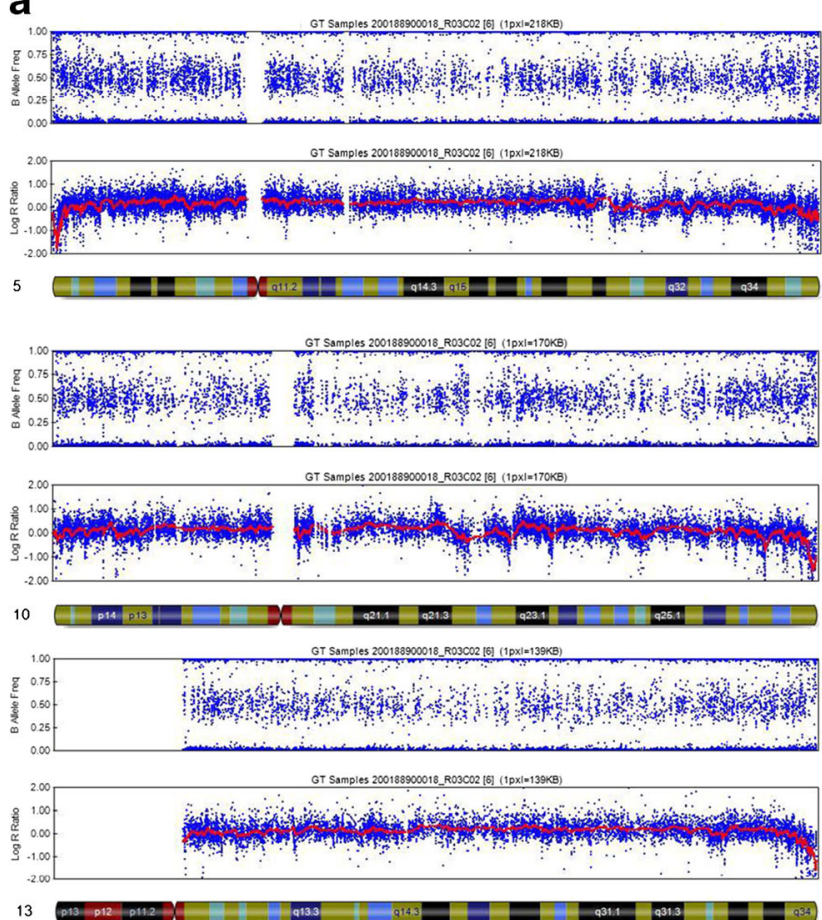

Fig. 2 Normal and abnormal molecular karyotypes of trophectoderm cells (TE) from blastocysts biopsied using MDA-based SNP microarray. a Normal diploid diagnostic reading obtained from TE for Chromosomes (Chr.) 5, 10, and 13. b Deletion of p13.1 $\rightarrow$ pter reading of Chr. 5 combined with a duplication of $\mathrm{p} 12.1 \rightarrow$ pter reading of Chr. 10 , which came from the same blastocyst of a $46, \mathrm{XY}, \mathrm{t}(5 ; 10)(\mathrm{P} 15 ; \mathrm{P} 12)$ carrier couple. $\mathrm{AA}, \mathrm{AB}$, and $\mathrm{BB}$ alleles were observed in $\mathrm{p} 13.1 \rightarrow$ qter of Chr. 5 and from p12.1 $\rightarrow$ qter of Chr. 10. However, AA and BB alleles were detected without $\mathrm{AB}$ in p13.1 $\rightarrow$ pter of $\mathrm{Chr}$. 5 , along with AAA,

than 5 megabases $(\mathrm{Mb})$ can be distinguished from the vast majority of unbalanced segregations. A specific abnormal product would not be scored if small copy number of variants or polymorphisms were under this cutoff value. The mean size of the chromosomal unbalance detected was up to $49 \mathrm{Mb}$ for duplication errors and $35 \mathrm{Mb}$ for deletion errors [28].

Another limitation was the use of MDA, which has a relatively high preferential amplification and allele dropout (ADO) rate (average of $25 \%$ ) [29], particularly in regions close to centromeres and telomeres [30]. Furthermore, some specific chromosomal regions, such as $1 \mathrm{q} 42,4 \mathrm{q} 35$, and $6 \mathrm{p} 25$, were reported to show a loss of representation after MDA [31]. All these detrimental factors might confound technicians when making a diagnosis. However, outcomes from studies

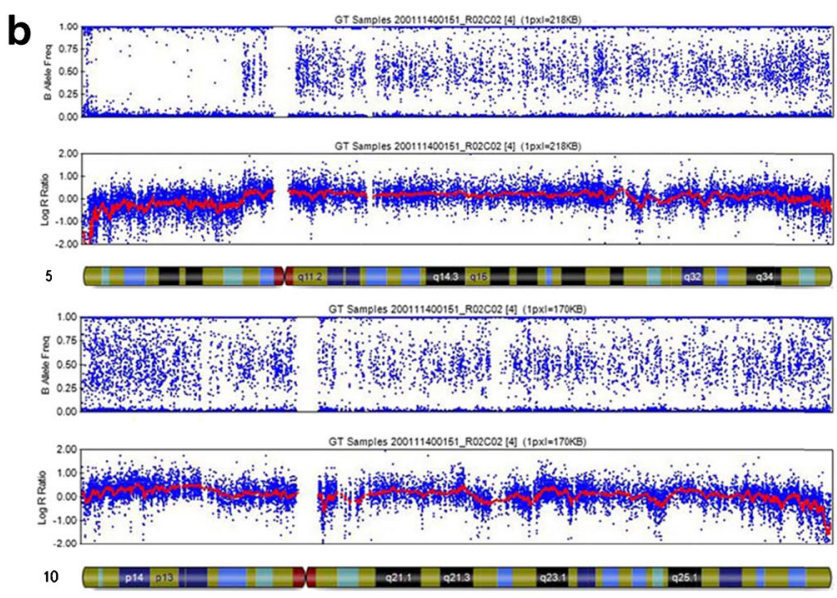

C

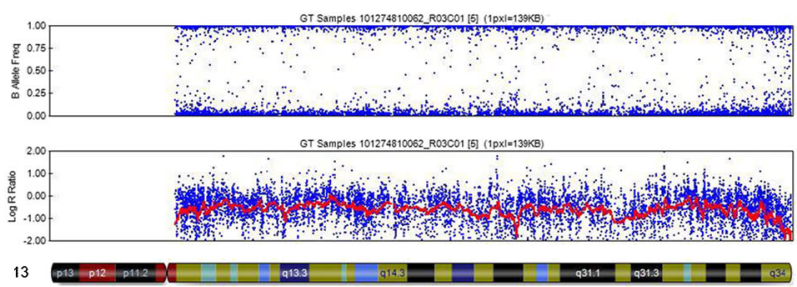

d

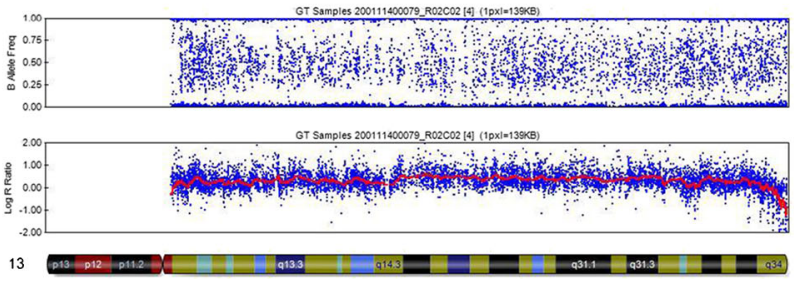

$\mathrm{AAB}$, and $\mathrm{BBB}$ from $\mathrm{p} 12.1 \rightarrow$ pter of Chr. 10 . A significant shift in the smooth $\log \mathrm{R}$ ratio is shown in $\mathrm{p} 13.1 \rightarrow$ pter of $\mathrm{Chr} .5$ and $\mathrm{p} 12.1 \rightarrow$ pter of Chr. 10. c Monosomy reading of Chr. 13. AA and BB alleles are illustrated without $A B$ alleles represented. A significant shift in the smooth $\log \mathrm{R}$ ratio was detected, consistent with the monosomy karyotype. d Trisomy reading of $\mathrm{Chr}$. 13. AAA, AAB, ABB, and BBB are shown without the $A B$ alleles represented. A significant shift in the smooth $\log \mathrm{R}$ ratio was observed, consistent with the trisomy karyotype

have shown these disadvantages do not have a determinative role. In our center, the preferential use of nanoliter reactors and analysis of multiple loci (STRs, short tandem repeats) might help to ameliorate amplification bias [29, 32]. The failure rate was decreased to $0.002 \%$ when three STRs markers were placed either side of the translocation breakpoints [33]. Furthermore, this platform provided an authentic diagnosis in other PGD centers, similar to that in our center [13, 18, 34].

\section{Conclusion}

To increase the likelihood of obtaining at least one normal/ balanced embryo following PGD by SNP microarray, we 
recommend that for females aged younger than 37 years with a basal FSH level under 11.4 IU/L, the number of blastocysts biopsied should be no less than 2 for RT carriers and no less than 4 for rcp. The accumulation of embryos before biopsy is a feasible strategy for patients with a limited number of embryos available for PGD. The outcome of our study might be more persuasive if more cases with a single transferrable embryo after diagnosis were available for analysis.

Acknowledgments The authors thank Prof. Fang Qun, Prof. Zhou Yi, and Dr. Lin Shaobin in the Fetal Medical Center for their help in providing desirable cord blood sample and chip results conducted in their platform. We also thank Mrs Wan Saihong for her help with inputting the data for analysis.

Author contributions Conceived and designed the experiments: YWX YZW. Performed the experiments: CHD YZW JW WZ YHZ. Analyzed the data: YZW YWX JW. Contributed reagents/materials/analysis tools: YWX YZW JW WZ RL. Wrote the paper: YZW YWX MFD CQZ.

\section{Compliance with ethical standards}

Funding statement This work was sponsored by Special Research Grant for Non-profit Public Service (Grant No. 201402004, Recipient YWX), Guangdong province science and technology plan project (Grant No. 2016A02021800, Recipient YWX), National Natural Science Foundation Project (Grant No. 81370765, Recipient CQZ), and Science and Information Technology of Guangzhou (Grant No. 201300000097, Recipient CQZ).

Conflict of interests The authors declare that they have no competing interests.

Open Access This article is distributed under the terms of the Creative Commons Attribution 4.0 International License (http:// creativecommons.org/licenses/by/4.0/), which permits unrestricted use, distribution, and reproduction in any medium, provided you give appropriate credit to the original author(s) and the source, provide a link to the Creative Commons license, and indicate if changes were made.

\section{References}

1. Alfarawati S, Fragouli E, Colls P, Wells D. First births after preimplantation genetic diagnosis of structural chromosome abnormalities using comparative genomic hybridization and microarray analysis. Human Reprod (Oxford, England). 2011;26(6):1560-74. doi:10.1093/humrep/der068.

2. Jin H, Ping L, Jie Q, Ying L, Yongjian C. Translocation chromosome karyotypes of the Robertsonian translocation carriers' embryos. Fertil Steril. 2010;93(4):1061-5. doi:10.1016/j. fertnstert.2008.11.020.

3. Ye Y, Qian Y, Xu C, Jin F. Meiotic segregation analysis of embryos from reciprocal translocation carriers in PGD cycles. Reprod Biomed Online. 2012;24(1):83-90. doi:10.1016/j. rbmo.2011.08.012.

4. Ozawa N, Maruyama T, Nagashima T, Ono M, Arase T, Ishimoto $\mathrm{H}$, et al. Pregnancy outcomes of reciprocal translocation carriers who have a history of repeated pregnancy loss. Fertil Steril. 2008;90(4):1301-4. doi:10.1016/j.fertnstert.2007.09.051.
5. Treff NR, Northrop LE, Kasabwala K, Su J, Levy B, Scott Jr RT. Single nucleotide polymorphism microarray-based concurrent screening of 24-chromosome aneuploidy and unbalanced translocations in preimplantation human embryos. Fertil Steril. 2011;95(5):1606-12.e1-2. doi:10.1016/j.fertnstert.2010.11.004.

6. Fiorentino F, Biricik A, Bono S, Spizzichino L, Cotroneo E, Cottone G, et al. Development and validation of a next-generation sequencing-based protocol for 24-chromosome aneuploidy screening of embryos. Fertil Steril. 2014;101(5):1375-82. doi:10.1016/j. fertnstert.2014.01.051.

7. Bono S, Biricik A, Spizzichino L, Nuccitelli A, Minasi MG, Greco E, et al. Validation of a semiconductor next-generation sequencingbased protocol for preimplantation genetic diagnosis of reciprocal translocations. Prenat Diagn. 2015;35(10):938-44. doi:10.1002 pd.4665.

8. Zhang W, Liu Y, Wang L, Wang H, Ma M, Xu M, et al. Clinical application of next-generation sequencing in preimplantation genetic diagnosis cycles for Robertsonian and reciprocal translocations. J Assist Reprod Genet. 2016;33(7):899-906. doi:10.1007/s10815016-0724-2.

9. Tan YQ, Tan K, Zhang SP, Gong F, Cheng DH, Xiong B, et al. Single-nucleotide polymorphism microarray-based preimplantation genetic diagnosis is likely to improve the clinical outcome for translocation carriers. Human Reprod (Oxford, England). 2013;28(9): 2581-92. doi:10.1093/humrep/det271.

10. Harper JC, Wilton L, Traeger-Synodinos J, Goossens V, Moutou C, SenGupta SB, et al. The ESHRE PGD Consortium: 10 years of data collection. Hum Reprod Update. 2012;18(3):234-47. doi:10.1093 /humupd/dmr052.

11. Moutou C, Goossens V, Coonen E, De Rycke M, Kokkali G, Renwick P, et al. ESHRE PGD Consortium data collection XII: cycles from January to December 2009 with pregnancy follow-up to October 2010. Hum Reprod (Oxford, England). 2014;29(5):880 903. doi:10.1093/humrep/deu012.

12. Chang EM, Han JE, Kwak IP, Lee WS, Yoon TK, Shim SH. Preimplantation genetic diagnosis for couples with a Robertsonian translocation: practical information for genetic counseling. J Assist Reprod Genet. 2012;29(1):67-75. doi:10.1007/s10815-011-9654-1.

13. Idowu D, Merrion K, Wemmer N, Mash JG, Pettersen B, Kijacic D, et al. Pregnancy outcomes following 24-chromosome preimplantation genetic diagnosis in couples with balanced reciprocal or Robertsonian translocations. Fertil Steril. 2015;103(4):1037-42. doi:10.1016/j.fertnstert.2014.12.118.

14. Demko ZP, Simon AL, McCoy RC, Petrov DA, Rabinowitz M. Effects of maternal age on euploidy rates in a large cohort of embryos analyzed with 24-chromosome single-nucleotide polymorphism-based preimplantation genetic screening. Fertil Steril. 2016;105(5):1307-13. doi:10.1016/j.fertnstert.2016.01.025.

15. Xu K, Montag M. New perspectives on embryo biopsy: not how, but when and why? Semin Reprod Med. 2012;30(4):259-66. doi:10.1055/s-0032-1313905.

16. Scott Jr RT, Upham KM, Forman EJ, Zhao T, Treff NR. Cleavagestage biopsy significantly impairs human embryonic implantation potential while blastocyst biopsy does not: a randomized and paired clinical trial. Fertil Steril. 2013;100(3):624-30. doi:10.1016/j. fertnstert.2013.04.039.

17. Cimadomo D, Capalbo A, Ubaldi FM, Scarica C, Palagiano A, Canipari R, et al. The impact of biopsy on human embryo developmental potential during preimplantation genetic diagnosis. Biomed Res Int. 2016;2016:7193075. doi:10.1155/2016/7193075.

18. Johnson DS, Gemelos G, Baner J, Ryan A, Cinnioglu C, Banjevic $\mathrm{M}$, et al. Preclinical validation of a microarray method for full molecular karyotyping of blastomeres in a 24-h protocol. Hum Reprod (Oxford, England). 2010;25(4):1066-75. doi:10.1093 /humrep/dep452. 
19. Colls P, Escudero T, Fischer J, Cekleniak NA, Ben-Ozer S, Meyer $\mathrm{B}$, et al. Validation of array comparative genome hybridization for diagnosis of translocations in preimplantation human embryos. Reprod Biomed Online. 2012;24(6):621-9. doi:10.1016/j. rbmo.2012.02.006.

20. Kroener L, Ambartsumyan G, Briton-Jones C, Dumesic D, Surrey $\mathrm{M}$, Munne S, et al. The effect of timing of embryonic progression on chromosomal abnormality. Fertil Steril. 2012;98(4):876-80. doi:10.1016/j.fertnstert.2012.06.014.

21. Taylor TH, Patrick JL, Gitlin SA, Wilson JM, Crain JL, Griffin DK. Comparison of aneuploidy, pregnancy and live birth rates between day 5 and day 6 blastocysts. Reprod Biomed Online. 2014;29(3): 305-10. doi:10.1016/j.rbmo.2014.06.001.

22. Su Y, Li JJ, Wang C, Haddad G, Wang WH. Aneuploidy analysis in day 7 human blastocysts produced by in vitro fertilization. Reprod Biol Endocrinol RB\&E. 2016;14:20. doi:10.1186/s12958-016-0157-x.

23. Xiong B, Tan K, Tan YQ, Gong F, Zhang SP, Lu CF, et al. Using SNP array to identify aneuploidy and segmental imbalance in translocation carriers. Genomics Data. 2014;2:92-5. doi:10.1016/j. gdata.2014.05.007.

24. Chatziparasidou A, Nijs M, Moisidou M, Chara O, Ioakeimidou C, Pappas C, et al. Accumulation of oocytes and/or embryos by vitrification: a new strategy for managing poor responder patients undergoing pre implantation diagnosis. F1000Research. 2013;2:240. doi: 10.12688/f1000research.2-240.v2.

25. Tulay P, Gultomruk M, Findikli N, Bahceci M. Number of embryos biopsied as a predictive indicator for the outcome of preimplantation genetic diagnosis by fluorescence in situ hybridisation in translocation cases. Zygote (Cambridge, England). 2015:1-8. doi: 10.1017/s0967199414000793.

26. Scriven PN, Flinter FA, Khalaf Y, Lashwood A, Mackie OC. Benefits and drawbacks of preimplantation genetic diagnosis (PGD) for reciprocal translocations: lessons from a prospective cohort study. Eur J Human Genet EJHG. 2013;21(10):1035-41. doi:10.1038/ejhg.2013.9.

27. Tobler KJ, Brezina PR, Benner AT, Du L, Xu X, Kearns WG. Two different microarray technologies for preimplantation genetic diagnosis and screening, due to reciprocal translocation imbalances, demonstrate equivalent euploidy and clinical pregnancy rates. J Assist Reprod Genet. 2014;31(7):843-50. doi:10.1007/s10815014-0230-3.

28. Li G, Jin H, Xin Z, Su Y, Brezina PR, Benner AT, et al. Increased IVF pregnancy rates after microarray preimplantation genetic diagnosis due to parental translocations. Syst Biol Reprod Med. 2014;60(2):119-24. doi:10.3109/19396368.2013.875241.

29. Harton GL, De Rycke M, Fiorentino F, Moutou C, SenGupta S, Traeger-Synodinos J, et al. ESHRE PGD consortium best practice guidelines for amplification-based PGD. Human Reprod (Oxford, England). 2011;26(1):33-40. doi:10.1093/humrep/deq231.

30. Arriola E, Lambros MB, Jones C, Dexter T, Mackay A, Tan DS, et al. Evaluation of Phi29-based whole-genome amplification for microarray-based comparative genomic hybridisation. Lab Invest $\mathrm{J}$ Tech Methods Pathol. 2007;87(1):75-83. doi:10.1038 /labinvest.3700495.

31. Iwamoto K, Bundo M, Ueda J, Nakano Y, Ukai W, Hashimoto E, et al. Detection of chromosomal structural alterations in single cells by SNP arrays: a systematic survey of amplification bias and optimized workflow. PLoS One. 2007;2(12):e1306. doi:10.1371 /journal.pone.0001306.

32. Marcy Y, Ishoey T, Lasken RS, Stockwell TB, Walenz BP, Halpern AL, et al. Nanoliter reactors improve multiple displacement amplification of genomes from single cells. PLoS Genet. 2007;3(9): 1702-8. doi:10.1371/journal.pgen.0030155.

33. Fiorentino F, Kokkali G, Biricik A, Stavrou D, Ismailoglu B, De Palma R, et al. Polymerase chain reaction-based detection of chromosomal imbalances on embryos: the evolution of preimplantation genetic diagnosis for chromosomal translocations. Fertility and sterility. 2010;94(6):2001-11, 11.e1-6. doi: 10.1016/j. fertnstert.2009.12.063.

34. Li G, He N, Jin H, Liu Y, Guo Y, Su Y, et al. The Influence of Single Nucleotide Polymorphism Microarray-Based Molecular Karyotype on Preimplantation Embryonic Development Potential. PLoS One. 2015;10(9), e0138234. doi:10.1371/journal.pone.0138234. 